\title{
Investigation of the Effect of Additive White Noise on the Dynamics of Contact Interaction of the Beam Structure
}

\author{
Olga Saltykova ${ }^{1}$, Alexander Krechin ${ }^{1}$ \\ olga_a_saltykova@mail.ru| san9.antonov@yandex.ru \\ ${ }^{1}$ Yuri Gagarin State Technical UniversityofSaratov, Saratov, Russia
}

The purpose of this work is to study and scientific visualization the effect of additive white noise on the nonlinear dynamics of beam structure contact interaction, where beams obey the kinematic hypotheses of the first and second approximation. When constructing a mathematical model, geometric nonlinearity according to the T. von Karman model and constructive nonlinearity are taken into account. The beam structure is under the influence of an external alternating load, as well as in the field of additive white noise. The chaotic dynamics and synchronization of the contact interaction of two beams is investigated. The resulting system of partial differential equations is reduced to a Cauchy problem by the finite difference method and then solved by the fourth order Runge-Kutta method.

Keywords: nonlinear dynamics, contact interaction, chaotic phase synchronization, white noise.

\section{Introduction}

The mechanics of contact interaction is one of the most rapidly developing topics of the mechanics of a deformable solid and is widely used in various fields of science [2, 4, 5]. A mathematical model of the contact interaction of two beams, described by the kinematic hypotheses of the first and second approximations [1], was constructed. An external alternating load and a white noise field affect one of the beams. Using the means of scientific visualization of the results of mathematical modeling, the nonlinear dynamics of the contact interaction of the beam structure located in the field of additive white noise is studied.

\section{Statement of the problem}

Geometric nonlinearity of beams was adopted according to the model of T. von Karman, the contact interaction is described by the B.Ya.Kantor model [3]. The equations of motion, boundary and initial conditions are obtained from the HamiltonOstrogradsky energy principle. Beam 1 obeys the kinematic hypothesis of the first approximation (Euler-Bernoulli model) under the action of transversal load and white noise, beam 2 is described by the kinematic hypothesis of the second approximation (Timoshenko model). The study of nonlinear dynamics is based on the study of phase portraits, wavelet and Fourier spectra, signals, chaotic phase synchronization, Lyapunov indicators. The values of the highest Lyapunov exponent are calculated by three methods: using the Kantz, Wolf and Rosenstein algorithm.

The equations of beams motion will take the form:

$$
\left\{\begin{array}{l}
\frac{1}{\lambda^{2}}\left\{F_{2}\left(w_{i}, w_{i}\right)+F_{1}\left(u_{i}, w_{i}\right)-\frac{1}{12} \frac{\partial^{4} w_{1}}{\partial x^{4}}\right\}-\frac{\partial^{2} w_{1}}{\partial t^{2}}-\varepsilon_{1} \frac{\partial w_{1}}{\partial t}+ \\
+(-1)^{i} K\left(w_{1}-w_{2}-h_{k}\right) \Psi+q(x, t)=0, \\
\frac{\partial^{2} u_{1}}{\partial x^{2}}+F_{3}\left(w_{i}, w_{i}\right)-\frac{\partial^{2} u_{1}}{\partial t^{2}}=0 ; \\
\frac{1}{3}\left(\frac{\partial^{2} w_{2}}{\partial x^{2}}+\frac{\partial \gamma_{x 2}}{\partial x}\right)+\frac{1}{\lambda^{2}}\left(L_{1}\left(w_{i}, u_{i}\right)+\frac{3}{2} L_{2}\left(w_{i}, w_{i}\right)+L_{3}\left(w_{i}, u_{i}\right)\right)+ \\
+(-1)^{i} K\left(w_{1}-w_{2}-h_{k}\right) \Psi-\frac{\partial^{2} w_{2}}{\partial t^{2}}-\varepsilon_{1} \frac{\partial w_{2}}{\partial t}=0 ; \\
\frac{\partial^{2} u_{2}}{\partial x^{2}}+L_{4}\left(w_{i}, w_{i}\right)-\frac{\partial^{2} u_{2}}{\partial t^{2}}=0 ; \\
\frac{\partial^{2} \gamma_{x 2}}{\partial x^{2}}-8 \lambda^{2}\left(\frac{\partial w_{2}}{\partial x}+\gamma_{x 2}\right)-\frac{\partial^{2} \gamma_{x 2}}{\partial t^{2}}=0 ; i=1,2,
\end{array}\right.
$$

$$
\begin{aligned}
& i=1,2 \quad-\quad \text { are serial number of beams. } \\
& F_{1}\left(u_{i}, w_{i}\right)=\frac{\partial^{2} u_{i}}{\partial x^{2}} \frac{\partial w_{i}}{\partial x}+\frac{\partial u_{i}}{\partial x} \frac{\partial^{2} w_{i}}{\partial x^{2}}, \\
& F_{2}\left(w_{i}, w_{i}\right)=\frac{3}{2} \frac{\partial^{2} w_{i}}{\partial x^{2}}\left(\frac{\partial w_{i}}{\partial x}\right)^{2}, F_{3}\left(w_{i}, w_{i}\right)=\frac{\partial^{2} w_{i}}{\partial x^{2}} \frac{\partial w_{i}}{\partial x}, \\
& L_{1}\left(w_{i}, u_{i}\right)=\frac{\partial^{2} w_{i}}{\partial x^{2}} \frac{\partial u_{i}}{\partial x}, L_{2}\left(w_{i}, w_{i}\right)=\frac{\partial^{2} w_{i}}{\partial x^{2}}\left(\frac{\partial w_{i}}{\partial x}\right)^{2}, \\
& L_{3}\left(w_{i}, u_{i}\right)=\frac{\partial w_{i}}{\partial x} \frac{\partial^{2} u_{i}}{\partial x^{2}}, L_{4}\left(w_{i}, w_{i}\right)=\frac{\partial w_{i}}{\partial x} \frac{\partial^{2} w_{i}}{\partial x^{2}} \quad \text { are the }
\end{aligned}
$$

nonlinear operators, $\gamma_{x i}$-is lateral shift function, $w_{i}, u_{i}$-are functions of deflection and displacement of beams, respectively, $K$ - stiffness coefficient of transversal compression of the structure in the contact zone, $h_{k}$ - the gap between the beams, the thickness of the beams $b=1, \varepsilon_{1}$ - damping coefficient, $\lambda=\frac{a}{(2 h)}$ - beam geometry parameter.

The boundary conditions in the case of rigid pinching and the initial conditions should be added to equations (1).

For the beam described by the hypothesis of the first approximation, the boundary conditions (2) and the initial conditions (3):

$$
\begin{aligned}
& w_{i}(0, t)=w_{i}(1, t)=u_{i}(0, t)= \\
& =u_{i}(1, t)=\frac{\partial w_{i}(0, t)}{\partial x}=\frac{\partial w_{i}(1, t)}{\partial x}=0 . \\
& w_{i}(x)_{\mid t=0}=0, u_{i}(x)_{\mid t=0}=0, \\
& \frac{\partial w_{i}(x)}{\partial t}=0,\left.\frac{\partial u_{i}(x)}{\partial t}\right|_{\mid t=0}=0 .
\end{aligned}
$$

For the beam described by the hypothesis of the second approximation, the boundary conditions (4) and the initial conditions (5):

$$
\begin{aligned}
& w(0, t)=w(1, t)=0 ; u(0, t)=u(1, t)=0 ; \\
& \gamma_{x}(0, t)=\gamma_{x}(1, t)=0 ; \frac{\partial w(0, t)}{\partial x}=\frac{\partial w(1, t)}{\partial x}=0 ; \\
& w(x, t)_{\mid t=0} 0, u(x, t)_{\mid t=0}=0, \gamma_{x}(x, t)_{\mid t=0}=0,
\end{aligned}
$$

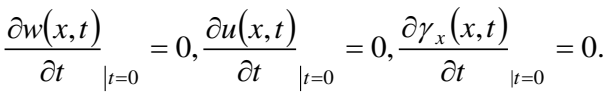

Beam 1 is affected by a distributed transverse alternating load of the form, additive white noise is added to the system of equations in the form of a random term with constant intensity $P n=P n_{0}\left(2.0 * \operatorname{rand}() /(65535+1.0), \quad P n_{0}-\right.$ is the noise intensity; $\operatorname{rand}()$ - standard $\mathrm{C}++$ function that accepts a random 
integer value from 0 to 65535 . This model was calculated using a program written in $\mathrm{C}++$. Visualization and analysis of the results was carried out on the basis of the MathCad and MATLAB programs.

$$
q=q_{0} \sin (\omega t)+P n,
$$

where $\omega$ - is load frequency; $q$ - is load amplitude; $P n$ random term with constant intensity. The resulting system of partial differential equations is reduced to an Ordinary Differential Equation system by the finite difference method with a second-order approximation. The obtained Cauchy problem is solved by the Runge-Kutta method.

\section{Results of a numerical experiment}

We present the results of a study of the nonlinear dynamics of contact interaction of a beam structure in a white noise field, where beam 1 is described by the Euler - Bernoulli hypothesis, beam 2 is subject to Timoshenko's hypothesis (Fig. 1).

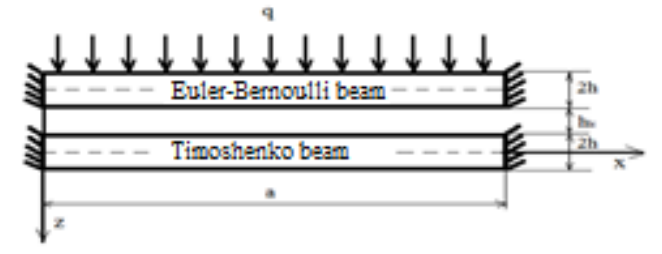

Figure 1. Beams structure

Table 1

Dynamic characteristics of beams $\lambda=50, h_{k}=0,1, q_{0}=1400, P_{n 0}=0$

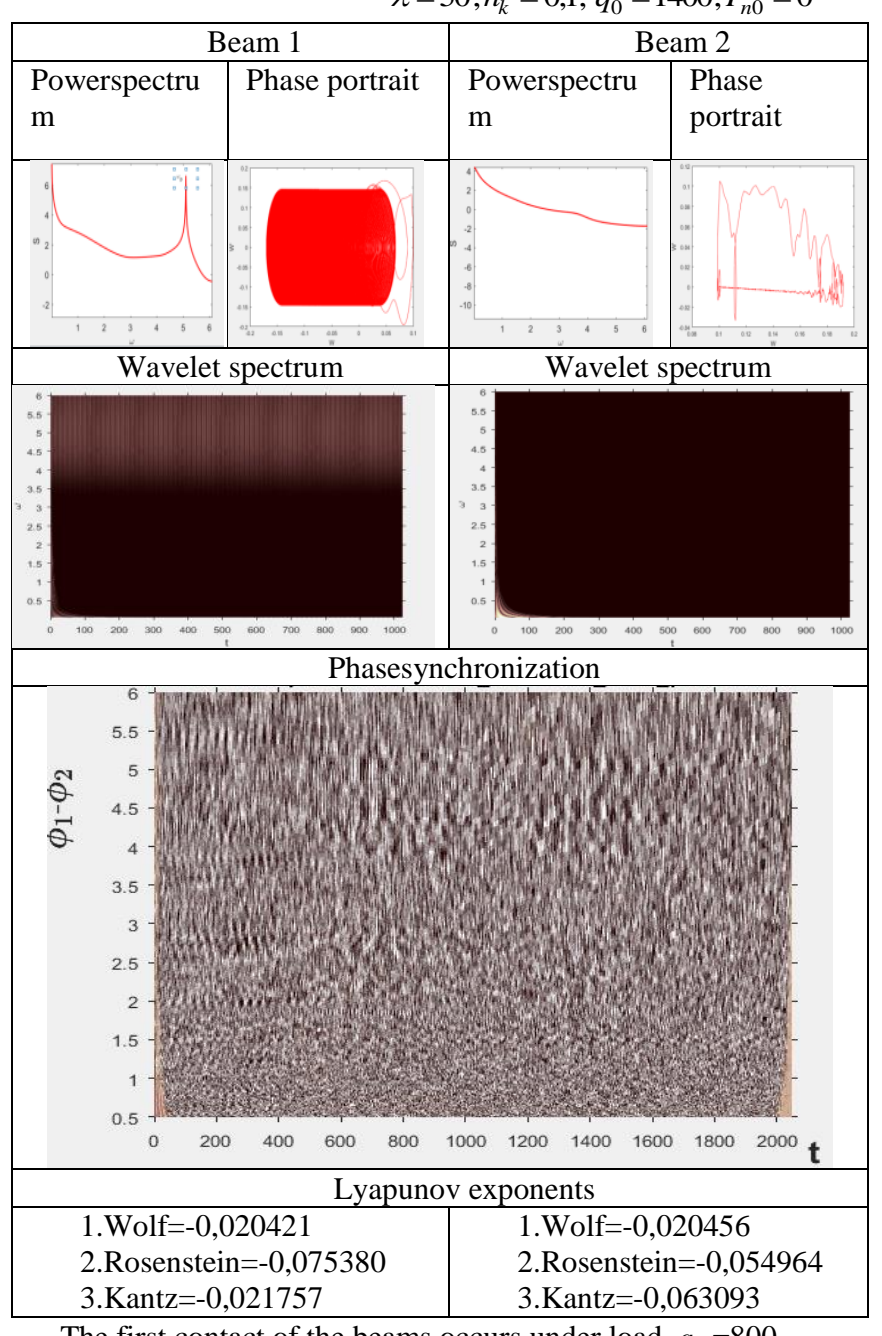

The first contact of the beams occurs under load $q_{0}=800$.
Increasing the load to 1400 (Table 1) leads to a change in the frequencies of the beams, on the power spectrum of the beam 1 there is one frequency: $\omega_{p}=5,1$. On the power spectrum of beam 2 , there are no pronounced frequencies.

The oscillations of the system at a given load are harmonic, chaos is not observed, as evidenced by phase portraits, wavelet spectra portrait of phase synchronization, as well as Lyapunov exponents, calculated by three different methods (Wolf, Rosenstein, Kantz) are negative.

Table 2

Dynamic characteristics of beams

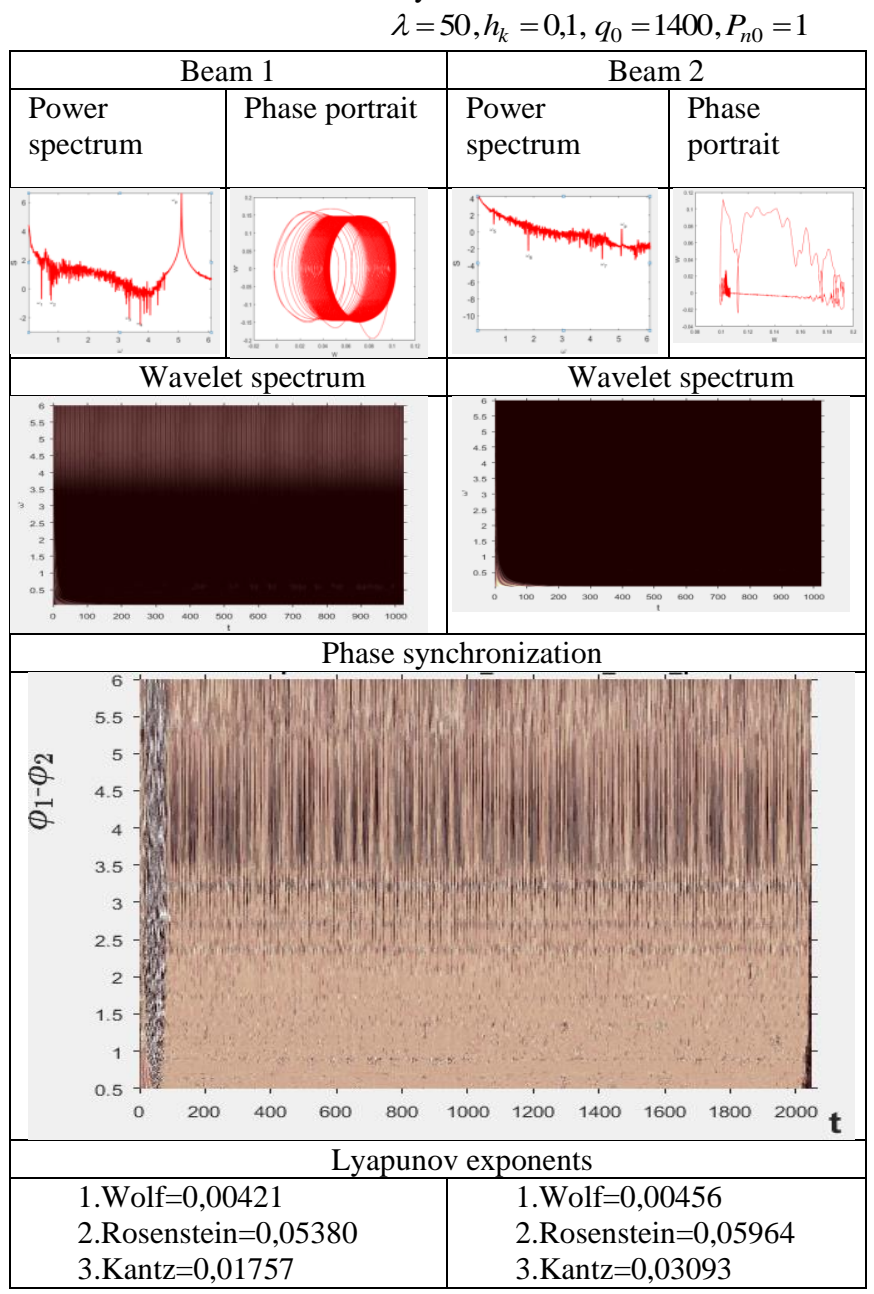

When adding a noise component (Table 2), the dynamics of the structure changes.

The power spectrum of beam 1 contains five frequencies.:

$\omega_{1}=\frac{\omega_{p}}{11}, \omega_{2}=0.757, \omega_{3}=\frac{4 \omega_{p}}{6}, \omega_{4}=\frac{5 \omega_{p}}{11}, \omega_{p}=5,1$. Two

frequencies $\omega_{2}, \omega_{p}{ }^{-}$are linear independent, and other frequencies are their linear combinations.

The power spectrum of beam 2 contains four frequencies.: $\omega_{5}=\frac{12 \omega_{p}}{100}, \omega_{6}=1.82, \omega_{7}=\frac{14 \omega_{p}}{16}, \omega_{p}=5,1$. Two $\quad$ frequencies $\omega_{6}, \omega_{p}$-are linear independent, and other frequencies are their linear combinations.

When adding a noise component, the system went into a chaotic state, which is visible in the wavelet spectra and in the phase synchronization portrait, as well as in Lyapunov's indicators.

The transition of the system to chaos occurred through the scenario of Ruel-Takens-Newhouse. 
Table 3

Dynamic characteristics of beams $\lambda=50, h_{k}=0,1, q_{0}=55000, P_{n 0}=0$

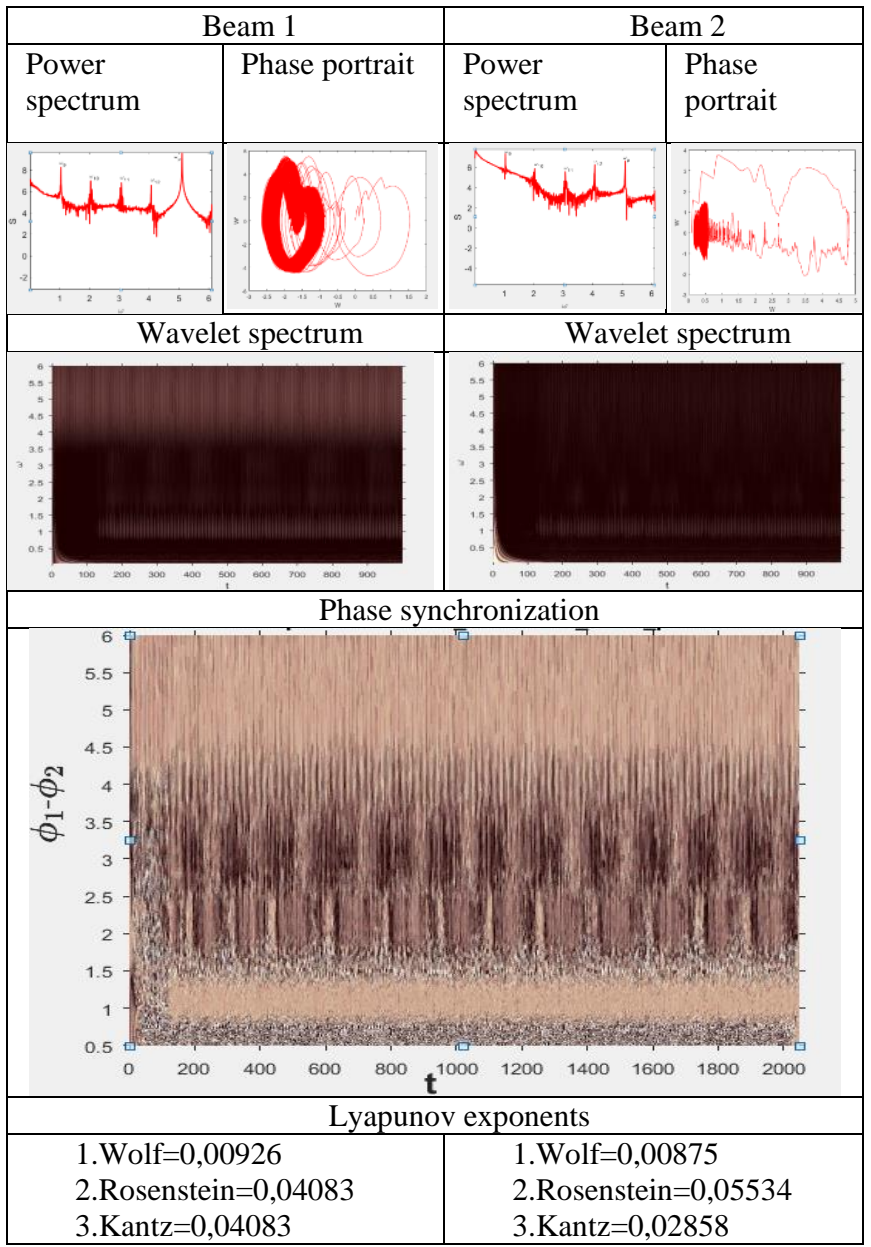

At $q_{0}=55000$ (Table 3 ) the power spectrums of beam 1 and beam 2 contains five frequencies: $\omega_{10}=\frac{\omega_{p}}{5}, \omega_{11}=2.12, \omega_{12}=\frac{3 \omega_{p}}{5}, \omega_{13}=2\left(\omega_{p}-\omega_{3}\right), \omega_{p}=5,1$.

At $q_{0}=55000, \mathrm{t}>100$ frequency synchronization occurs: $\omega_{1}, \omega_{2}, \omega_{p}$.

With an increase in the amplitude of the forced oscillations, the character of the beam signals changes from quasi-periodic to chaotic.

We can observe the scenario of Ruel-Takens-Newhouse. Wavelet spectra visualization allow you to see the change in the nature of oscillations of beams in time.

In Table 4 , when adding white noise $P_{n o}=1$, visual, and therefore qualitative changes in the dynamics of the model were not detected.

The power spectrums of beam 1 and beam 2 contains five frequencies described above.

Note that in this case the influence of the noise load practically did not affect the nonlinear dynamics of the contact interaction of the beams.

An increase in the amplitude of white noise does not lead to a change in the scenario of transition of oscillations into chaotic.

In Tables 5 and 6, we compare the Fourier spectra and signals without a white noise field and with noise, respectively.
Table 4

Dynamic characteristics of beams $\lambda=50, h_{k}=0,1, q_{0}=55000, P_{n 0}=1$

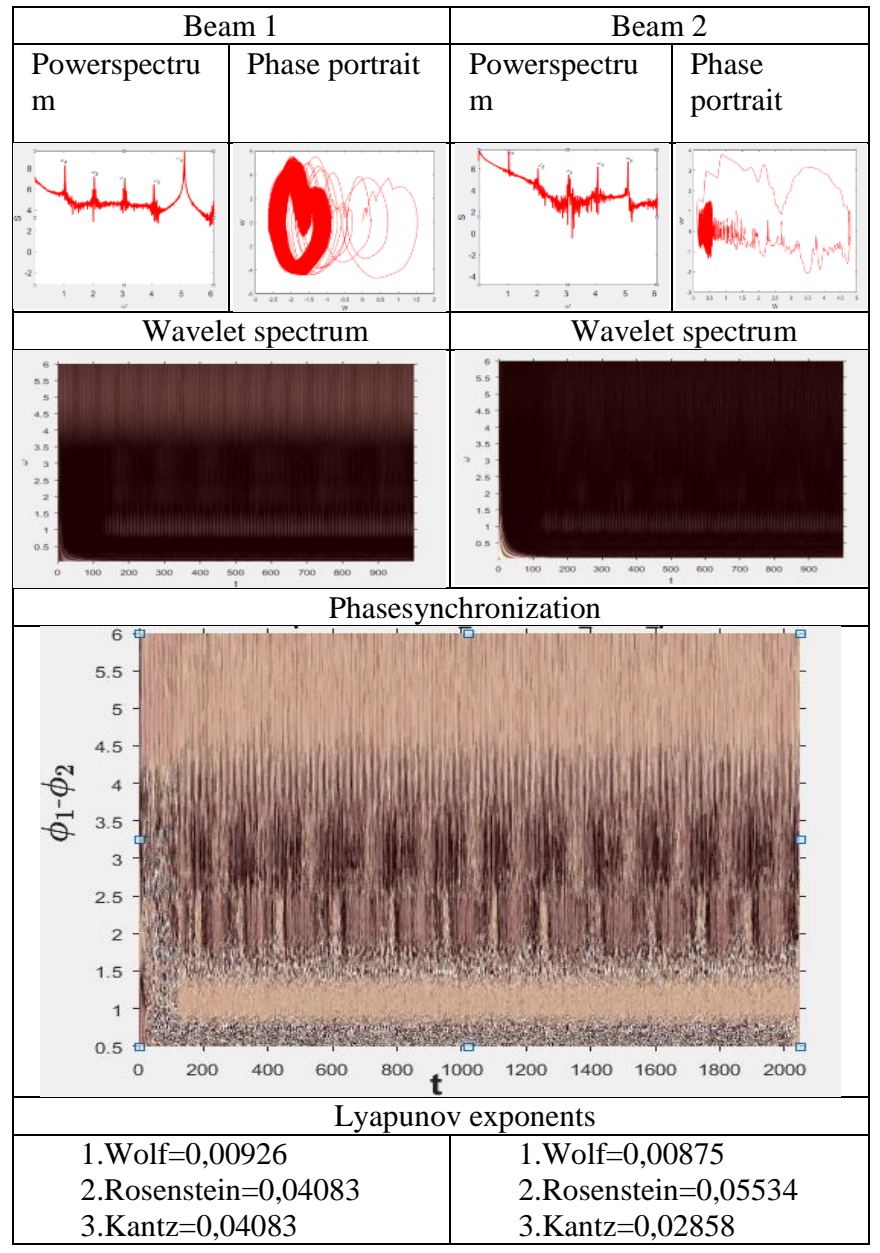

Table 5

Dynamic characteristics of beams $\lambda=50, h_{k}=0,1, q_{0}=1400$

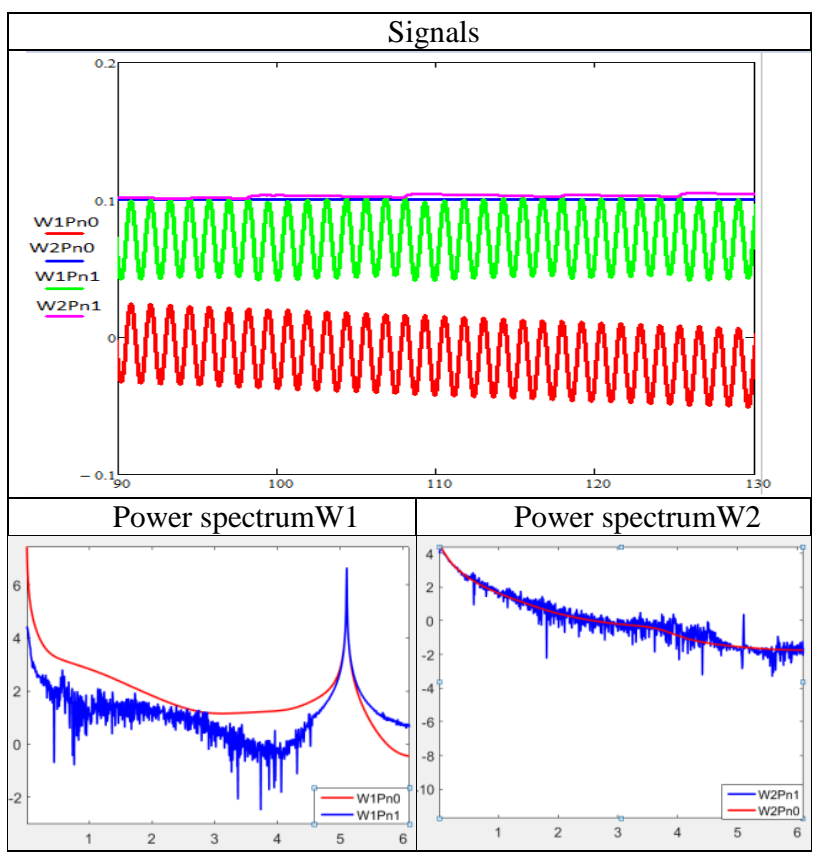


Table6

Dynamic characteristics of beams $\lambda=50, h_{k}=0,1, q_{0}=55000$

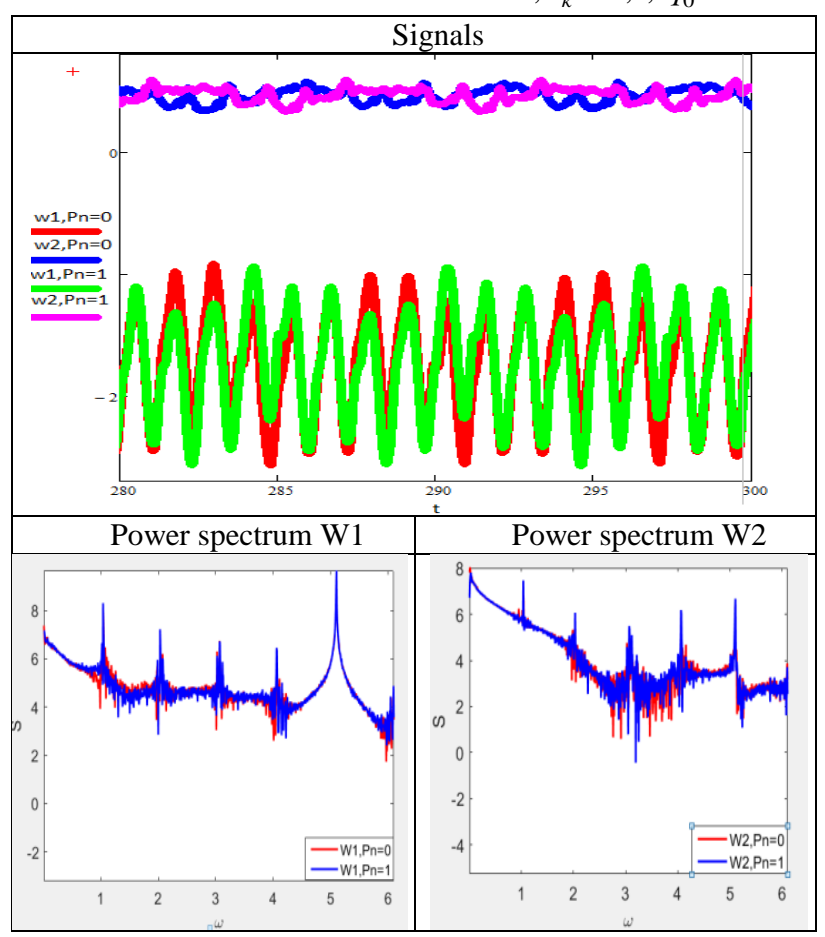

Visualization of signals and power spectra allows to visually see (Table 5 and Table 6 ) the qualitative changes in the vibrations of the beam structure, under the influence of an external alternating load of different intensity and white noise.

\section{Conclusion}

A mathematical model of the contact interaction of two geometrically non-linear beams, described by the kinematic hypotheses of the first and second approximation, is constructed. Data visualization made it possible to compare signals, phase synchronization, phase portraits and identify features of the dynamics of contact interaction of the studied beam structure. One of the structure beams is under the influence of an external distributed alternating load and in the field of white additive noise. The effect of the intensity of the noise component $\left(P_{n}\right)$ on the amplitude-frequency characteristics of the beams was investigated. A numerical experiment was performed for $P_{n}=$ $0.1 ; 0.5 ; 1$, with the same characteristics of the external alternating load. With small amplitudes of forcing vibrations $\left(\mathrm{q}_{0}<10000\right)$, the presence of additive white noise with intensity $P_{n}=1$ significantly changes the nonlinear dynamics of the structure under study and leads to a transition of system oscillations from harmonic to chaotic. When $P_{n}=0.1 ; 0.5$ the influence of white noise is not significant and can be neglected. At $\mathrm{q}_{0}>12000$, the effect of additive white noise is less obvious. This is due to the fact that the system is already in a chaotic state. The influence of additive white noise on the scenario of transition from harmonic to chaotic oscillations is investigated. Using scientific data visualization shown it is shown that the consideration of the noise component does not affect the scenario of transition of oscillations to chaotic ones. The transition to chaotic oscillations occurs according to the scenario of RuelTakens- Newhouse. The phenomenon of a decrease in the noise component under the action of additive white noise was found (Table 6).

\section{Acknowledgments}

This work was supported by the grant of the Russian Science Foundation16-11-10138.

\section{References}

[1] Awrejcewicz, J., Krysko, A.V., Pavlov, S.P., Zhigalov, M.V., \&Krysko, V.A. (2017). Chaotic dynamics of size dependent Timoshenko beams with functionally graded properties along their thickness. Mechanical Systems and Signal Processing, 93, 415-430.

[2] Awrejcewicz, J., Krysko-Jr, V.A., Yakovleva, T.V., Krysko, V.A. (2016). Noisy contact interactions of multi-layer mechanical structures coupled by boundary conditions. Journal of Sound and Vibration, 369, 77-86.

[3] Kantor B.Ya. Contact problems of the nonlinear theory of shells of revolution, Kiev, Naukova Dumka, 1991, p. 136

[4] Krysko, V.A., Awrejcewicz, J., Papkova, I.V., Saltykova, O.A., Krysko, A.V. (2019). Chaotic Contact Dynamics of Two Microbeams under Various Kinematic Hypotheses. International Journal of Nonlinear Sciences and Numerical Simulation, 20(34), 373-386.

[5] Yakovleva, T.V., Krysko Jr, V.A., \&Krysko, V.A. (2019, March). Nonlinear dynamics of the contact interaction of a threelayer plate-beam nanostructure in a white noise field. In Journal of Physics: Conference Series (Vol. 1210, No. 1, p. 012160). IOP Publishing. 